\title{
EDITORIAL CIENCIA Y SOCIEDAD. UNA REVISTA CON NUEVOS RETOS
}

Cómo citar: Ulloa Hung, J. (2021). EDITORIAL: Ciencia y Sociedad. Una revista con nuevos retos. Ciencia y Sociedad, 46(1), 3-6. https://doi.org/10.22206/cys.2021.v46i1.pp3-6

Ciencia y Sociedad inicia el 2021 con actualizaciones en su alcance y estructura, cuyo objetivo es mejorar su calidad y declarar de manera explícita un enfoque exclusivamente orientado hacia la difusión científica. Los ajustes también incluyen aspectos de orden formal en función de adecuar el cuerpo informativo de la revista y hacer más dinámico el flujo tradicional de sus procesos de recepción y revisión de colaboraciones, así como propiciar mayor información sobre nuestras normas editoriales y otros requisitos imprescindibles para los envíos.

La redefinición del alcance de Ciencia y Sociedad a partir del 2021 la orientan hacia un énfasis en la trandicisplinariedad y multidisciplinariedad, a fin de contribuir con la apertura, el redimensionamiento y la amplitud del rol de las Ciencias Sociales y las Humanidades, demanda actual, necesaria e imprescindible, en las naciones de América Latina y el Caribe. Desde esta perspectiva sus objetivos estarán mayormente concentrados en difundir investigaciones en Ciencias Sociales y Humanidades, que fomenten el abordaje de problemáticas sociales y culturales a partir de la diversidad de enfoques y las sinergias entre disciplinas científicas.

En función de esa orientación, Ciencia y Sociedad pretende ampliar los espacios de diálogo, interacción y, especialmente, de debate, entre diferentes líneas de investigación y discursos teóricos. Su finalidad esencial se centrará en mostrar una noción más amplia, diversa y compleja de las Ciencias Sociales y las Humanidades a partir de la adopción de perspectivas trans y multidisciplinarias.

Una redefinición de sus secciones se encuentra a tono con este nuevo énfasis, encaminado a que la revista también se trasforme en espacio de discusión cientifica. La nueva estructura incluye tres secciones. En primer lugar, la sección de artículos, esencialmente reservada para textos originales relacionados con resultados científicos obtenidos desde investigaciones empíricas o análisis teóricos y filosóficos que contribuyan al avance en el conocimiento o 
las discusiones sobre un tema. Estas contribuciones deben ofrecer avances en la investigación, caracterizar o definir tendencias en un área específica del conocimiento, proponer nuevos enfoques teóricos o metodológicos, nuevas hipótesis, o proporcionar ideas o percepciones provocativas $\mathrm{y}$ bien fundamentadas sobre una problemática.

En segundo lugar, un apartado para comentarios, el cual pretende erigirse en espacio de debate y discusión sobre cuestiones sociales o humanísticas relevantes y, especialmente, en un contexto para comentar, analizar, o responder a artículos y textos publicados en Ciencia y Sociedad. Además, ser una sección para publicar colaboraciones que constituyan comentarios sobre cualquier artículo previamente publicado, lo que puede incluir la respuesta del autor o autora a comentarios realizados. Esto no solo pretende fomentar la discusión científica, sino también contribuir aún más a la calidad y profundidad de los trabajos que publica la revista.

Por último, las reseñas de libros constituyen la tercera sección dentro de la nueva estructura de Ciencia y Sociedad. Esta recogerá colaboraciones que analicen, comenten o reseñen nuevas publicaciones dentro del campo de las Ciencias Sociales y las Humanidades o sus relaciones con otras ramas del conocimiento. Se trata de un espacio para mantener al corriente a la comunidad científica y de lectores de la revista sobre literatura importante en campos y disciplinas que cubre su enfoque temático.

Como hasta el momento, Ciencia y Sociedad mantendrá su Editorial que introduce cada número y destaca aspectos relevantes que merecen atención de los lectores.

Otro elemento que ha sido ajustado es la proyección temática de Ciencia y Sociedad como revista internacional revisada por pares. En ese sentido, los artículos resultados de investigaciones, las reseñas de libros o los comentarios se circunscribirán a los aportes vinculados con disciplinas como las Ciencias políticas; Ciencias de la comunicación; Antropología; Historia;
Sociología; Filosofía; Psicología; Estudios de Género; así como sobre las relaciones entre cultura, sociedad, tecnología y ambiente.

Invitamos a lectores y autores a revisar cambios y ajustes en nuestra política editorial e instrucciones para autores a través de nuestra plataforma de revista científica.

Desde el punto de vista de su contenido, este primer número de Ciencia y Sociedad del 2021 concentra colaboraciones en tres de sus líneas temáticas: los estudios de género, las ciencias de la comunicación, y la psicología.

El tema de género se enfoca a través de una problemática que constituye un flagelo en la sociedad contemporánea, especialmente en naciones de América Latina y el Caribe, la violencia de género. Esta problemática se aborda a partir de una de sus aristas más espinosas, el hostigamiento y el acoso sexual.

Manejado con una intención esencialmente preventiva, que toma como escenario los centros de educación superior en México, el artículo que abre este número resalta el rol del género como un indicador importante al momento de perpetuar o padecer hostigamiento sexual en las universidades.

Desde un abordaje que transita de lo general a lo particular, esta colaboración se centra en describir los mecanismos empleados en universidades públicas estatales mexicanas para intervenir en casos de violencia de género y analiza el conocimiento de las acciones diseñadas para prevenir, atender y erradicar el hostigamiento sexual. Con perspectiva descriptiva y usando instrumentos de investigación como escala Likert el estudio demuestra como las potenciales víctimas de esta forma de violencia desconocen los mecanismos de denuncia, aspecto, que, asociado a otros factores, contribuyen en la invisibilización y en el manejo de esta problemática.

Un segundo artículo, desarrollado como ensayo crítico reflexivo, centra sus esfuerzos en el análisis de la comunicación científica y procura demostrar que las fuentes 
de la subjetividad en este tipo de discurso no están en las marcas que plasma el investigador, sino en el rigor y claridad del mismo. En ese sentido, se analiza lo inevitable de la subjetividad en algunas etapas del proceso de producción científica, y, por tanto, el hecho de la cancelación del sujeto de la investigación o su aparición gramatical solo eventual, como una limitación sin fundamentos reales. Basado en una metodología y sustentación teórica proveniente del análisis crítico del discurso y la filosofía de las ciencias, este artículo sostiene que el uso del sujeto en el discurso científico en contextos adecuados no solo favorece su claridad, sino que tampoco afecta la objetividad. Desde esa perspectiva, se enfrasca en develar que, en el discurso científico, al igual que en todos los discursos, se manifiestan multiplicidad de voces, y por tanto manejar la voz pertinente en función de su claridad y objetividad debe ser parte de las competencias pragmáticas y científicas de un investigador.

El análisis del discurso con enfoque filosófico constituye el centro de otra de las colaboraciones presentes en esa edición de Ciencia y Sociedad. Este se materializa a través del análisis de la obra de Francisco Moscoso Puello, intelectual dominicano con una vida dedicada a las ciencias y las letras cuya producción literaria se enmarca en la primera mitad del siglo xx. El análisis de la obra de este autor se concentra en dos aspectos esenciales, su visión de la identidad, y su concepción sobre la raza. Ambos elementos son contextualizados a luz del momento histórico en que este produce su obra, las influencias de las teorías científicas imperantes, así como su propia trayectoria personal.

A partir de una metodología que tiene como fundamento el método hermenéutico, el artículo nos devela las relaciones entre el corpus ideológico de Moscoso Puello y la manifestación o expresión de su fatalismo o angustia social de ser mulato presente en sus obras. En ese orden, también es capaz de poner al descubierto cómo ese fenómeno ha continuado latente como una alternativa ideológica en función de negar la negritud y como pretexto para exaltar una hispanidad imaginaria y tradicionalista.
Por último, la Psicología está presente en el corpus de artículos de esta entrega de la revista a través del análisis de aspectos prácticos y epistemológicos vinculados a la psicología ocupacional. Utilizando un enfoque empírico inductivo y una estrategia de revisión bibliográfica fundamentada en el análisis crítico de enunciados teóricos y autores, esta colaboración enmarca algunas de las problemáticas esenciales de esta rama de la psicología en el contexto del neoliberalismo cultural contemporáneo. Se trata de un artículo de revisión bibliográfica cuyo eje central es el reclamo de un carácter crítico y pluridisciplinario en el abordaje conceptual y metodológico de la realidad organizacional. Aspecto que además es considerado un elemento de peso para diversificar la disciplina y sacarla de los estigmas que solo la vinculan profesionalmente con los designios ideológicos del gerencialismo.

Un elemento interesante de esta revisión teórica es que la misma concluye enunciando y evaluando lo que el autor considera desafíos esenciales de la psicología frente a ese encasillamiento en prácticas gerenciales en función de la eficiencia, o como parte de la virtud dentro de la narrativa empresarial contemporánea. Aspectos que incitan al debate y la apertura reflexiva sobre los ejes vertebradores de la psicología organizacional, fenómeno que precisamente se encuentra a tono con lo que se propone Ciencia y Sociedad a partir de este nuevo desafío como publicación científica.

Finalmente, esta primera edición de Ciencia y Sociedad del 2021 cierra con la reseńa de una obra enmarcada en los estudios patrimoniales y la trascendencia de este aspecto para comprender las historias y culturas de las actuales naciones caribeñas. La reseña de Cuba: Arqueología y legado histórico exalta como la tendencia a la multidisciplinariedad en función de reconocer la diversidad étnica y cultural se va imponiendo y cobra cada vez mayor auge dentro los estudios antropológicos y arqueológicos, y en general en los estudios del pasado. Tendencia que no solo 
obedece al necesario reclamo de creadores y actuales portadores de ciertos legados históricos, sino a la imprescindible comprensión de la historia desde la multivocalidad como eje articulador de la descoloni- zación de nuestro pensamiento y nuestras acciones, en la que la multiplicidad de enfoques, las colaboraciones y las sinergias de investigación deben ser la norma y no la excepción.

\section{Dr. Jorge Ulloa Hung}

Profesor Investigador del Instituto Tecnológico de

Santo Domingo (INTEC)

Director de Ciencia y Sociedad

Correo-e: jorge.ulloa@intec.edu.do

Página web: https://www.intec.edu.do 